\title{
Vorwort der Herausgeber
}

\author{
Timo Schmid • Markus Zwick
}

(C) Springer-Verlag GmbH Germany, part of Springer Nature 2018

Liebe Leserinnen und Leser,

nach einem heißen Sommer liegt nun das Heft 2 des Bands 12 des AStA Wirtschaftsund Sozialstatistisches Archiv (WiSoStA) auch in der Papierform vor. Im Gegensatz zum letzten Jahr hat sich die Anzahl der Einreichungen nun doch spürbar erhöht. Mit diesem Heft liegen Ihnen vier interessante und relevante Beiträge vor und auch für die kommenden Hefte sind derzeit schon eine Reihe Aufsätze im Prozess der Evaluierung. Nichtsdestotrotz ist die Akquise von Beiträgen eine permanente Aufgabe eines jeden Herausgebers. Aus diesem Grund auch die erneute Thematisierung innerhalb dieses Editorials. Wie wir in einem früheren Heft berichtet haben, sind wir auch an universitären Abschlussarbeiten in gekürzter Form für das WiSoStA interessiert, so sie Themenbereiche dieser Zeitschrift berührt werden. Bisher haben uns solche Beiträge leider noch nicht erreicht. Es wäre sehr schön, wenn Sie solche Einreichungen in Ihrem universitären Umfeld mit unterstützen könnten und angehende Wissenschaftlerinnen und Wissenschaftler auf die Möglichkeit einer Publikation im WiSoStA hinweisen könnten. Wir sind bestrebt solche Einreichungen im Begutachtungsprozess besonders zu berücksichtigen. Dies heißt nicht, dass Beiträge des wissenschaftlichen Nachwuchses anderen Qualitätsanforderungen unterliegen werden, sondern, dass die Gutachten auch eine gewisse didaktische Qualität aufweisen werden.

T. Schmid $(\bowtie)$

Institut für Statistik und Ökonometrie, Freie Universität Berlin, Berlin, Deutschland

E-Mail: Timo.Schmid@fu-berlin.de

M. Zwick

Institut für Forschung und Entwicklung in der Bundesstatistik, Statistisches Bundesamt, Wiesbaden, Deutschland 
Aber nun zu den Ausführungen in diesem Heft. Den Anfang macht Andreas Kladroba et al. (2018) mit dem Beitrag ,Technologien statt Branchen: Eine Neuauswertung der FuE-Erhebung 2015“. Forschung und Entwicklung in den Unternehmen sind gewichtige Treiber des ökonomischen Wachstums in Deutschland. Aus diesem Grund kommt der Erhebung zur Forschung und Entwicklung des Deutschen Stifterverbandes eine wichtige Rolle zu, da diese Daten auch im Rahmen von Förderprogrammen mit herangezogen werden. Da Förderungen mehr und mehr technologiegebunden vergeben werden, ist der Zusammenhang von Brachen und Technologien von Interesse. Die vorliegende Arbeit erläutert, wie durch eine Neuauswertung der Daten der FuE-Erhebung 2015 eine Zuordnung der unternehmensinternen FuE-Aufwendungen zu Technologien vorgenommen wurde.

Statistische Prozesskontrolle ist insbesondere im Bereich der Überwachung von Fertigungsprozessen bekannt. Robert Garthoff und Philipp Otto (2018) zeigen hingegen, wie die räumliche Prozesskontrolle zur Beschreibung der Bevölkerungsentwicklung in Deutschland angewandt werden kann. Sie bieten einen großen Überblick über die bestehende Literatur im Bereich der räumlichen Prozesskontrolle und erläutern detailliert ihr Verfahren zur Überwachung eines multivariaten räumlichen autoregressiven Prozesses. Mit Hilfe von Kontrollkarten illustrieren sie die Ergebnisse der statistischen Prozesskontrolle für die Landkreise und kreisfreie Städte in Deutschland.

Die Arbeitszeiten von Professorinnen und Professoren in Deutschland sind weniger reglementiert als in vielen anderen Berufen bzw. Bereichen. Führt dies jedoch zu einer höheren oder niedrigeren Wochenarbeitszeit im Vergleich zur üblichen $40 \mathrm{~h}$ Woche? Claus Weihs et al. (2018) untersuchen die Arbeitszeiten von vollzeitbeschäftigten Professorinnen und Professoren in Deutschland und kommen durchschnittlich auf eine Gesamtarbeitszeit von $56 \mathrm{~h}$ pro Woche. Zusätzlich betrachten sie auch die Arbeitszeiten für Teilaufgaben und stellen dabei eine Diskrepanz zwischen der Einschätzung der Gesamtarbeitszeit und der Summe der Arbeitszeiten von Teilaufgaben fest. Methodisch untersuchen sie die Fragestellung sowohl aus frequentistischer als auch aus Bayesianischer Sicht. Die Ergebnisse beruhen auf einer umfangreichen E-Mail Befragung von Professorinnen und Professoren in Deutschland, die kritisch mit anderen Studien in Vergleich gesetzt werden.

Auch in diesem Heft setzen wir die Reihe der Interviews mit namhaften Statistikern im WiSoStA fort. Dieses Mal mit Volker Mammitzsch, mit dem Walter Krämer (2018) während der Statistischen Woche 2016 in Augsburg die Zeit fand, das in diesem Heft veröffentlichte Interview zu führen. Wie viele der in dieser Reihe interviewten deutschen Statistikerinnen und Statistiker fand auch Volker Mammitzsch erst über Umwege zu der Wissenschaft. Da er in der DDR nicht zur Oberschule zugelassen war, machte er das Abitur erst nach einer Lehre, nachdem die Familie nach München gezogen war. Die Ausführungen dazu sind im Artikel sehr interessant beschrieben. Anschließend studierte er in München Mathematik und Physik und war mehrere Jahre an der LMU in verschiedenen Positionen tätig. Den Großteil seiner akademischen Karriere verbrachte Volker Mammitzsch, unterbrochen von verschiedenen Aufhalten u.a. an der Penn State University (USA), an der Philipps-Universität Marburg. In der deutschen Statistiker-Gemeinde wird Volker Mammitzsch als Vermittler zwischen klassischer und mathematischer Statistik sehr 
geschätzt und organisierte zahlreiche Jahrestagungen und Konferenzen. In Krämer (2018) gibt Volker Mammitzsch einen spannenden Einblick in seinen persönlichen Werdegang.

Nun wünschen wir Ihnen, liebe Leserinnen und Leser, viel Spaß bei der Lektüre der zweiten diesjährigen Ausgabe des WiSoStA.

Timo Schmid und Markus Zwick

\section{Literatur}

Garthoff R, Otto P (2018) Verfahren zur Überwachung räumlicher autoregressiver Prozesse mit externen Regressoren. AStA Wirtsch Sozialstat Arch. https://doi.org/10.1007/s11943-018-0224-1

Kladroba A, Kudic M, Friz K et al (2018) Technologien statt Branchen: Eine Neuauswertung der FuEErhebung 2015. AstA Wirtsch Sozialstat Arch. https://doi.org/10.1007/s11943-018-0226-Z

Krämer W (2018) Interview mit Volker Mammitzsch. AstA Wirtsch Sozialstat Arch. https://doi.org/10. 1007/s11943-018-0225-0

Weihs S, Rodríguez TH, Doeckel M et al (2018) Arbeitszeiten von Professorinnen und Professoren in Deutschland 2016. AstA Wirtsch Sozialstat Arch. https://doi.org/10.1007/s11943-018-0227-y 